\title{
LETTER
}

\section{The negative impact of comorbidities on the disease course of COVID-19}

\author{
Noémi Zádori ${ }^{1,2}$, Szilárd Váncsa ${ }^{1,2}$, Nelli Farkas ${ }^{1,3}$, Péter Hegyi ${ }^{1,2,4}$ and Bálint Erőss ${ }^{1,2^{*}}$ (1) on behalf of the KETLAK \\ Study Group
}

(c) 2020 Springer-Verlag GmbH Germany, part of Springer Nature

\section{Dear Editor,}

Coronavirus Disease 2019 (COVID-19) is a viral infection caused by severe acute respiratory syndrome coronavirus 2 (SARS-CoV-2) with a mortality rate of $3-7 \%$ [1]. The high mortality results from fulminant pneumonia leading to acute respiratory distress syndrome and multiple organ failure $[2,3]$. Initial reports suggest that comorbidities cause a more severe course of infection and a poorer prognosis $[4,5]$. Considering the fast spread and high mortality of COVID-19, it is necessary to understand the possible risk factors affecting its progression. We aimed to perform a systematic search to evaluate the potential role of all reported comorbidities on the disease course. Details of our report are provided in Supplementary file 1.

We searched MEDLINE, Embase, Cochrane Central Register of Controlled Trials, Web of Science, and Scopus between 01/01/2020 and 05/11/2020. The main outcomes were mortality, intensive care unit (ICU) admission and severity. Definitions of the investigated outcomes are available in Supplementary file 2, Table 2. Odds ratios (OR) with $95 \%$ confidence intervals $(\mathrm{CI})$ were calculated to objectify the association between comorbidities and the outcomes by the random-effects model. The study was registered on PROSPERO (CRD42020176781).

Of 33,987 records screened, 61 cohort studies with 31,089 (median 162; IQR: 103-338) patients were included in the meta-analysis. The overall mortality rate was $10.0 \%, 19.9 \%$ of patients needed intensive, while the reported severity was $24.0 \%$. Underlying chronic

\footnotetext{
*Correspondence: eross.balint@pte.hu

1 Institute for Translational Medicine, Medical School, University of Pécs, Pécs 7624, Hungary

Full author information is available at the end of the article
}

Members of the KETLAK Study Group are given in the

Acknowledgements section. kidney disease (OR:5.3 CI 3.2-8.7), cardiovascular disease (OR:4.7, CI 2.9-7.6), cerebrovascular disease (OR:3.9, CI 1.8-8.3), chronic obstructive pulmonary disease (OR:3.7, CI 2.7-5.1), hypertension (OR:2.7, CI 1.74.4), malignancy (OR:2.6, CI 1.5-4.3), diabetes (OR:2.5, CI 1.7-3.6) and immunodeficiency (OR:1.6, CI 1.0-2.5) were associated with increased risk of mortality. The analysis could not prove that Hepatitis B infection or chronic liver disease were associated with mortality due to the low number of participants with comorbidities (Fig. 1, Suppl. File 2. Figs. 4-13).

Patients with a history of cerebrovascular disease (OR:3.5, CI 1.9-6.5), chronic obstructive pulmonary disease (OR:2.2, CI 1.5-3.4), cardiovascular disease (OR:2.1, CI 1.5-3.0), hypertension (OR:1.9, CI 1.5-2.5), diabetes (OR:1.8, CI 1.3-2.5), malignancy (OR:1.7, CI 1.0-2.7) needed intensive care more often. The analysis could not prove that chronic liver disease, immunodeficiency, chronic kidney disease and hepatitis $B$ infection were associated with higher admission rate to ICU (Supp. File 1. Fig. 2.; Suppl. File 2. Figs. 4-13).

Results regarding severity are shown in Supplementary file 1, Fig. 3. Reports on comorbidities not eligible for meta-analysis are summarized in Supplementary file 2, Table 6.

In summary, cerebrovascular disease, chronic obstructive pulmonary disease, cardiovascular disease, hypertension, diabetes mellitus, malignancy are significant risk factors for poor clinical outcome in COVID-19. Our findings emphasize the critical role of comorbidities in the course of COVID-19. These results could be used for risk stratification of patients infected with SARS-CoV-2 and should be taken into consideration when establishing a prognostic tool. Although no specific treatment has 


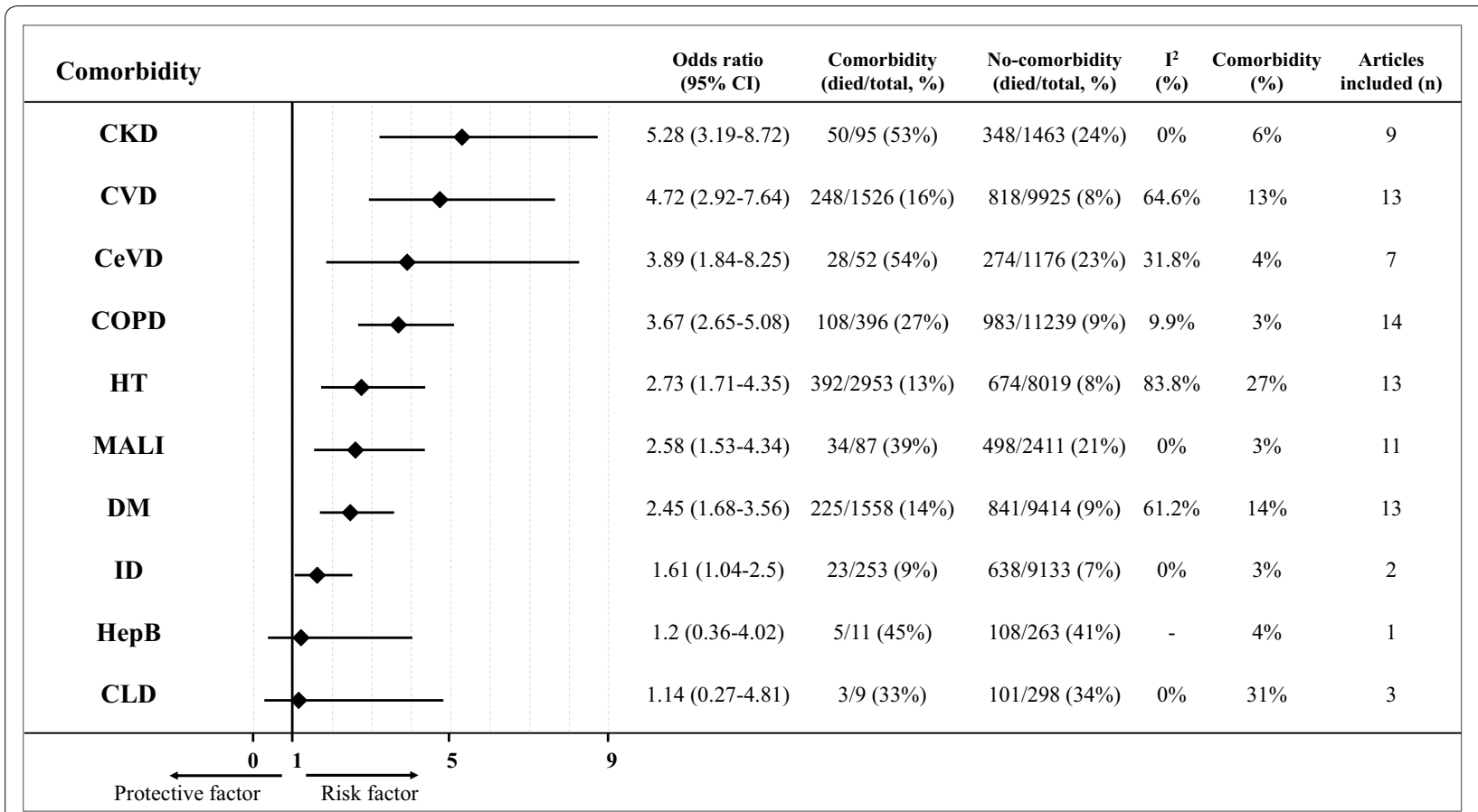

Fig. 1 Summary figure for odds ratios (OR) with 95\% confidence interval (95\% Cl) of mortality for different comorbidities. Forest plots with pooled ORs can be found in Suppl.File 2, Figs. 4-13. 95\% Cl confidence interval, CKD chronic kidney disease, CVD cardiovascular disease, CeVD cerebrovascular disease, COPD chronic obstructive pulmonary disease, HT hypertension, MALI malignancy, DM diabetes mellitus, ID immunodeficiency, HepB Hepatitis B, CLD chronic liver disease, 12 heterogeneity, $n$ number of articles included, OR odds ratio

been identified for COVID-19 yet, these results should be considered when applying prevention and therapy.

\section{Electronic supplementary material}

The online version of this article (https://doi.org/10.1007/s00134-020-06161-9) contains supplementary material, which is available to authorized users.

\section{Author details}

${ }^{1}$ Institute for Translational Medicine, Medical School, University of Pécs, Pécs 7624, Hungary. ${ }^{2}$ János Szentágothai Research Centre, University of Pécs, Pécs, Hungary. ${ }^{3}$ Institute of Bioanalysis, Medical School, University of Pécs, Pécs, Hungary. ${ }^{4}$ First Department of Medicine, University of Szeged, Szeged, Hungary.

\section{Acknowledgements}

The KETLAK Study Group:

Lajos Szakó: Institute for Translational Medicine, Medical School, University of Pécs, Pécs, Hungary and János Szentágothai Research Centre, University of Pécs, Pécs, Hungary. Nóra Vörhendi: Institute for Translational Medicine, Medical School, University of Pécs, Pécs, Hungary and János Szentágothai Research Centre, University of Pécs, Pécs, Hungary. Levente Frim: Institute for Translational Medicine, Medical School, University of Pécs, Pécs, Hungary. Zs. Réka Dömötör: Institute for Translational Medicine, Medical School, University of Pécs, Pécs, Hungary and University of Medicine, Pharmacy, Science and Technology of Targu Mures. Zsolt Szakács: Institute for Translational Medicine Medical School, University of Pécs, Pécs, Hungary and János Szentágothai Research Centre, University of Pécs, Pécs, Hungary. Szabolcs Kiss: Institute for Translational Medicine, Medical School, University of Pécs, Pécs, Hungary, First Department of Medicine, University of Szeged, Szeged, Hungary and Doctoral School of Clinical Medicine, University of Szeged, Szeged, Hungary. Mária Földi: Institute for Translational Medicine, Medical School, University of Pécs, Pécs, Hungary, First Department of Medicine, University of Szeged, Szeged, Hungary and Doctoral School of Clinical Medicine, University of Szeged, Sze- ged, Hungary. Fanni Dembrovszky: Institute for Translational Medicine, Medical School, University of Pécs, Pécs, Hungary and János Szentágothai Research Centre, University of Pécs, Pécs, Hungary. Marcell Imrei: Institute for Translational Medicine, Medical School, University of Pécs, Pécs, Hungary and János Szentágothai Research Centre, University of Pécs, Pécs, Hungary. Zsolt Molnár: Institute for Translational Medicine, Medical School, University of Pécs, Pécs, Hungary and Department of Anaesthesiology and Intensive Therapy, Poznan University for Medical Sciences, Poznan, Poland. Zoltán Péterfi: Division of Infectious Diseases, First Department of Medicine, Medical School, University of Pécs, Pécs, Hungary. Hussain Alizadeh: Division of Hematology, First Department of Medicine, Medical School, University of Pécs, Pécs, Hungary. László Czopf: Division of Cardiology, First Department of Medicine, Medical School, University of Pécs, Pécs, Hungary.

\section{Funding}

This work was funded by the Human Resources Development Operational Programme Grant, Grant Number: EFOP-3.6.2-16-2017-0006 - LIVE LONGER is co-financed by the European Union (European Regional Development Fund) within the framework of Programme Széchenyi 2020.

\section{Compliance with ethical standards}

\section{Conflict of interest}

The authors declare no conflict interests.

\section{Publisher's Note}

Springer Nature remains neutral with regard to jurisdictional claims in published maps and institutional affiliations.

Accepted: 12 June 2020

Published online: 29 June 2020 


\section{References}

1. World Health Organization. Coronavirus disease 2019 (COVID-19): situation report, 121. (2020). https://apps.who.int/iris/bitstream/handle/10665 /332156/nCoVsitrep20May2020-eng.pdf

2. Wu Z, McGoogan JM (2020) Characteristics of and important lessons from the coronavirus disease 2019 (COVID-19) outbreak in China: summary of a report of 72314 cases from the Chinese Center for Disease Control and Prevention. JAMA 323(13):1239-1242
3. Arentz M et al (2020) Characteristics and outcomes of 21 critically ill patients with COVID-19 in Washington State. JAMA 323(16):1612-1614. https://doi.org/10.1001/jama.2020.4326

4. Chen R et al. (2020) Risk factors of fatal outcome in hospitalized subjects with coronavirus disease 2019 from a nationwide analysis in China. Chest S0012-3692(20)30710-8.https://doi.org/10.1016/j.chest.2020.04.010

5. Guan W-J et al (2020) Clinical characteristics of coronavirus disease 2019 in China. N Engl J Med 382(18):1708-1720 